\title{
Molecular probing of low-temperature incommensurate phases
}

\author{
J. Kikas ${ }^{1}$, A. Suisalu ${ }^{2}$, A. Laisaar ${ }^{2}$, and An. Kuznetsov ${ }^{2}$ \\ ${ }^{1}$ Institute of Materials Science, University of Tartu, 4 Tähe, Tartu 51010, Estonia \\ E-mail: jaakk@physic.ut.ee \\ ${ }^{2}$ Institute of Physics, University of Tartu, 142 Riia, Tartu 51014, Estonia
}

\begin{abstract}
Two-dimensional (2D) excitation-emission spectra of biphenyl doped with free-base chlorin were measured at $5 \mathrm{~K}$ under various pressures up to $350 \mathrm{MPa}$. Besides the features related to zero-phonon lines and their phonon sidebands, a broad spectral band amounting to $80 \%$ of the total intensity at $5 \mathrm{~K}$ was revealed in the 2D spectra. The obtained inhomogeneous distribution function shows drastic changes with increasing pressure - the triplet structure observable at normal pressure in the incommensurate phase ICIII of biphenyl converges to a singlet in the high-pressure commensurate phase CI. These observations are assumed to reflect specific for incommensurate phases relaxation after an optical excitation of probe molecules and interaction of them with the incommensurate modulation wave.
\end{abstract}

PACS: 61.44.Fw, 62.50.+p, 64.70.Rh, 78.55.Kz

\section{Introduction}

Optical spectra of impurities in solid matrices [1] contain, in principle, rich information on both static structure and dynamical processes in solids. Even for strongly disordered materials the methods of site-selection spectroscopy, such as selective excitation $[2,3]$ and spectral hole burning [4,5], have revealed a lot of details otherwise obscured by the inhomogeneous broadening of conventional spectra. In this paper we make use of an advanced version of site-selection spectroscopy, namely two-dimensional (2D) excitation-emission spectroscopy (also called total luminescence spectroscopy [6]), in order to study local structure and dynamics in doped incommensurate solids. Incommensurate systems [7] are a specific and interesting class of solids showing a long-range order but lacking the translational lattice periodicity of crystals. In most systems exhibiting incommensurate behaviour, the incommensurate phase exists in quite a narrow temperature interval between an ordinary higher-temperature commensurate phase and a low-temperature lock-in phase, which is commensurate again, but generally has a larger unit cell. Incommensurate biphenyl is one of a few systems where such lock-in transition to a low-temperature commensurate structure has not been observed down to the lowest temperatures studied (60 mK [8], $70 \mathrm{mK}$ [9]). This makes it a suitable object of investigation using the methods of optical site-selection spectroscopy. (Note that some of the recent results on the temperature broadening and thermal cycling of spectral holes in doped biphenyl are presented in our papers $[8,10]$.) The phases of biphenyl and transitions between them have been extensively examined (see, e.g., [11] and references therein). By cooling a biphenyl sample it passes from the ordinary high-temperature crystalline phase CI to an incommensurate phase ICII at $40 \mathrm{~K}$ followed by transition to another incommensurate phase ICIII at $17 \mathrm{~K}$, the spatially modulated property being the twist angle between the planes of two phenyl rings. Under pressure at liquid helium temperature, the phase transitions ICIII $\rightarrow$ ICII and ICII $\rightarrow$ CI occur at $\sim 20 \mathrm{MPa}$ and $\sim 180 \mathrm{MPa}$, respectively [12]. As a possible reason for absence of the lock-in transition to a crystalline ground state at low temperatures, the metastability of the phase ICIII was proposed («incommensurate glass» [13]).

\section{Experimental}

An organic dye free base chlorin (7,8-dihydroporphin, $\mathrm{H}_{2}-\mathrm{C}_{20} \mathrm{~N}_{4} \mathrm{H}_{14}$ ) was used as a dopant in this study. A polycrystalline sample was obtained by slowly cooling the molten biphenyl down to the room temperature. Two-dimensional excitation-emission spectra were measured on a spectrometer, which consists of a tunable linear dye laser CR-490 (Coherent Inc.) and a double 
grating monochromator DFS-24 (LOMO) equipped with a CCD-camera DU420-BU (Andor). A small liquid-helium cryostat was used for measurements at 1 atm. For high-pressure measurements a system consisting of a 1.5-GPa helium gas compressor, optical high-pressure cell with sapphire windows and a large temperature-controlled liquid-helium cryostat was used.

\section{Results and discussion}

The two-dimensional excitation-emission spectrum (depicted as a plot of isointensity lines for emission at a constant excitation intensity) for chlorin-doped biphenyl in the incommensurate phase ICIII at $5 \mathrm{~K}$ and ambient pressure is shown in Fig. 1,a. Here the excitation was scanned by $0.02-\mathrm{nm}$ steps within the $(0-0)+863.4 \mathrm{~cm}^{-1}$ vibronic transition of the probe

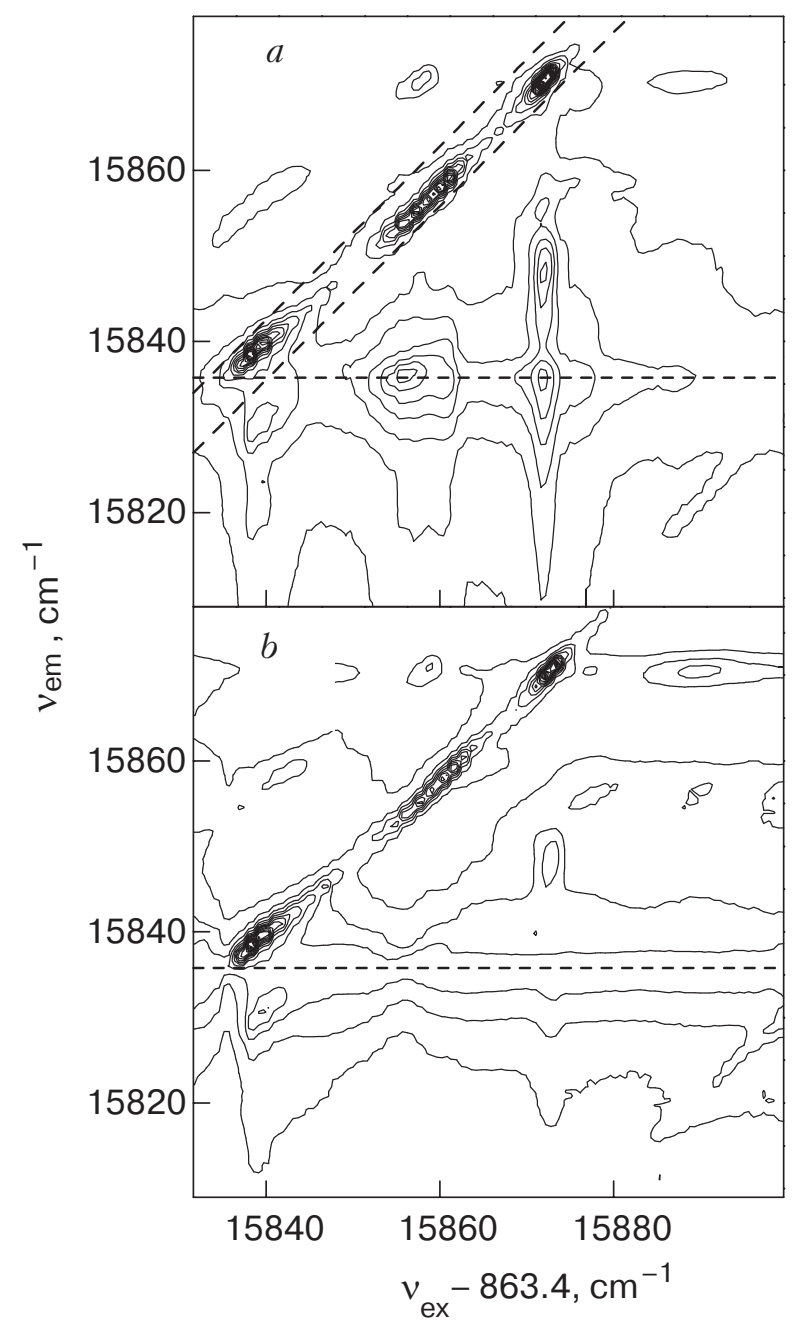

Fig. 1. Two-dimensional excitation-emission spectrum of chlorin-doped biphenyl at $5 \mathrm{~K}$ under normal pressure (incommensurate phase ICIII) ( $a$ ). The same but normalized to the intensity values along the horizontal dashed line in Fig. 1, $a(b)$. The dashed diagonal corridor represents the area used to determine the inhomogeneous distribution function depicted in Fig. 2,b.

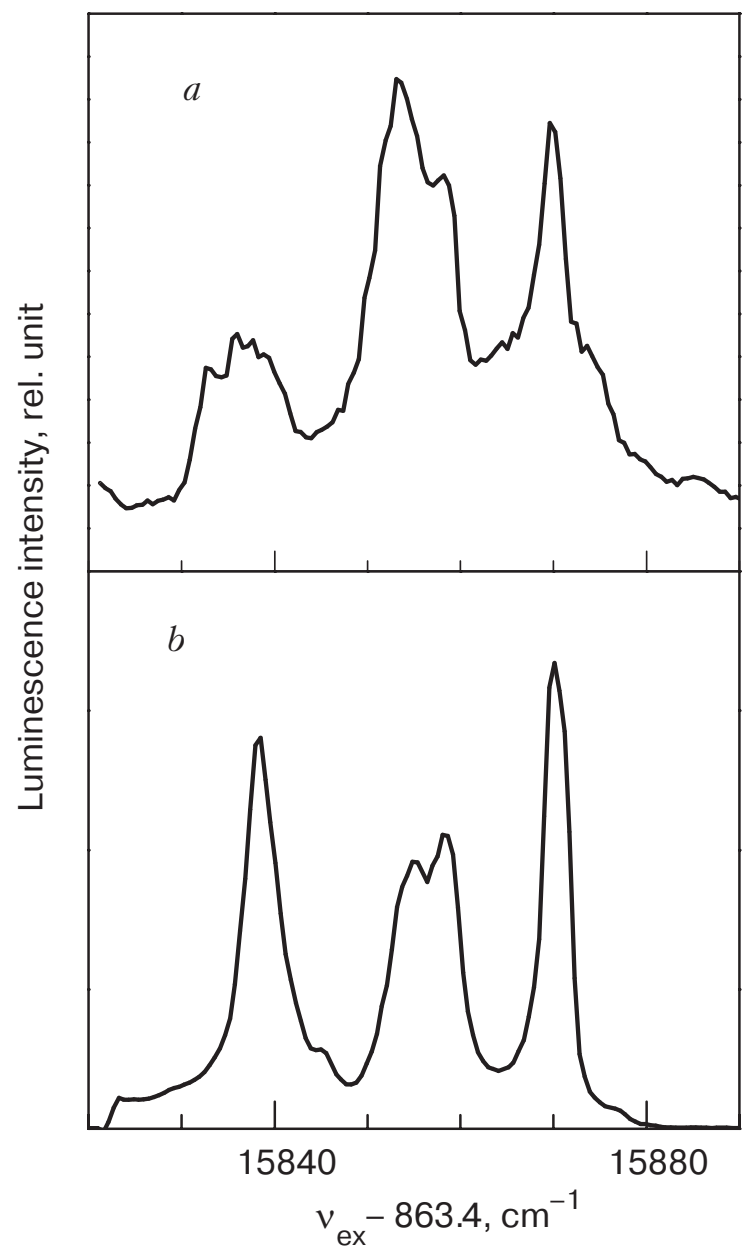

Fig. 2. Selective excitation spectrum of chlorin-doped biphenyl obtained from Fig. 1, $a$ by making a cut of 2D spectrum along the horizontal dashed line at $15836 \mathrm{~cm}^{-1}(a)$. The inhomogeneous distribution function obtained by making a diagonal cut of 2D spectrum in Fig. 1, $a(b)$.

molecule while the fluorescence emission was recorded in the $0-0$ region, $0-0$ denoting the resonant purely electronic transition $S_{0} \leftrightarrow S_{1}$. The high-intensity narrow «diagonal» features in the dashed corridor correspond to the three zero-phonon lines. By plotting the maximum emission intensity inside the corridor at each fixed excitation frequency, one obtains the static inhomogeneous distribution function (IDF) of probe transition frequencies as shown in Fig. 2,b. The shape of IDF reflects the distribution of static local environments around probe molecules. The triplet shape of IDF for chlorin in the ICIII phase of biphenyl can be interpreted as arising from interaction of a probe molecule with the static incommensurate modulation wave. While the outermost lines in the triplet can be attributed to edge singularities characteristic for such a situation [7], the middle peak may arise from pinning of the modulation wave by probe molecules. Such a viewpoint is further supported by the results of our 


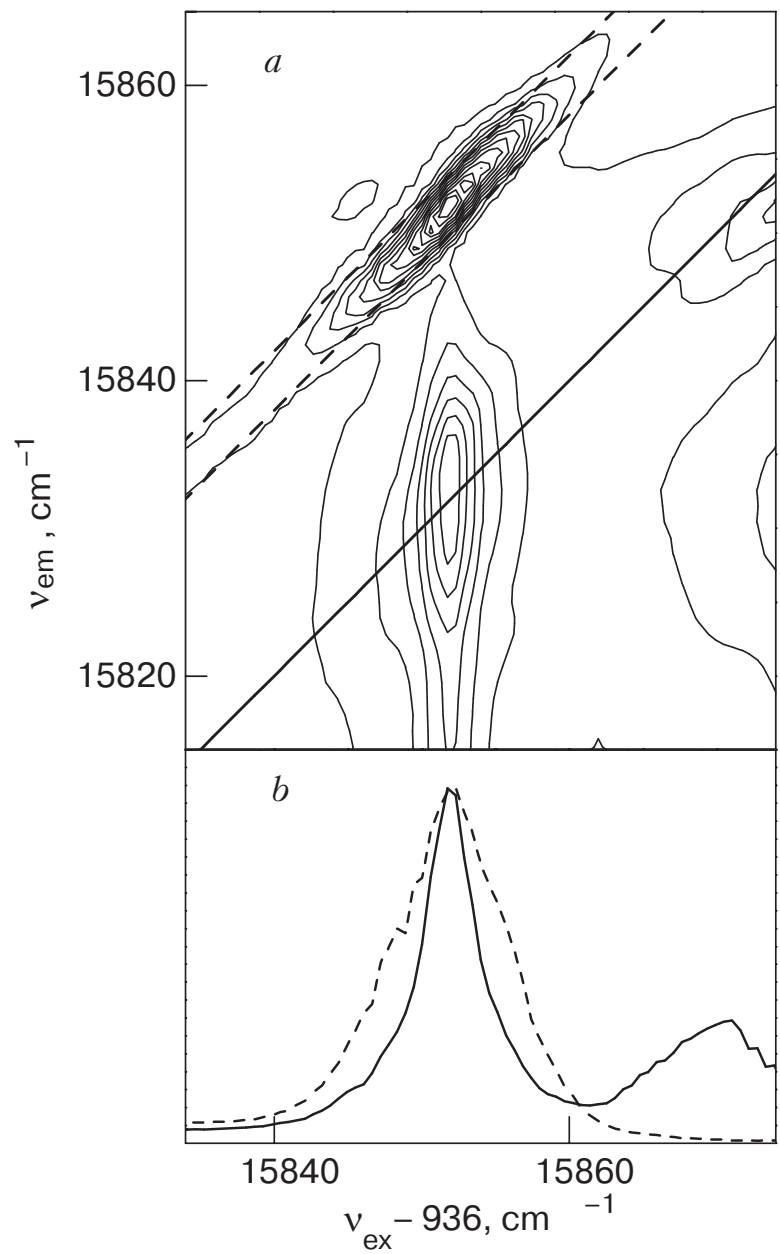

Fig. 3. Two-dimentional excitation-emission spectrum of chlorin-doped biphenyl at $5 \mathrm{~K}$ under pressure of $220 \mathrm{MPa}$ (crystalline phase CI) $(a)$. The respective inhomogeneous distribution function (dashed curve) and a diagonal cut (solid curve) along the solid line in Fig. 3, $a(b)$.

high-pressure measurements (Fig. 3), where one observes convergence of the low-pressure triplet to a high-pressure singlet at pressures above $180 \mathrm{MPa}$, i.e. in the crystalline phase CI (for more detailed discussion of pressure effects, see [12]). This proves that we have only one substitutional site for dopant molecules in the phase CI.

A surprising and remarkable feature in Fig. 1,a, however, is the existence of a broad 2D band located around the horizontal dashed line, which makes up more than $80 \%$ of the total intensity. As can be seen from Fig. 1,a, the position of the maximum of the broad-band emission (as indicated by the dashed line at $15836 \mathrm{~cm}^{-1}$ ) does not depend on the excitation frequency. Note also that this maximum is red-shifted from the red peak of IDF (see the vertical dashed line in Fig. 4), and therefore the broad band cannot be the phonon sideband of the red peak in IDF. The excitation spectrum of the broad 2D band, recorded selec-

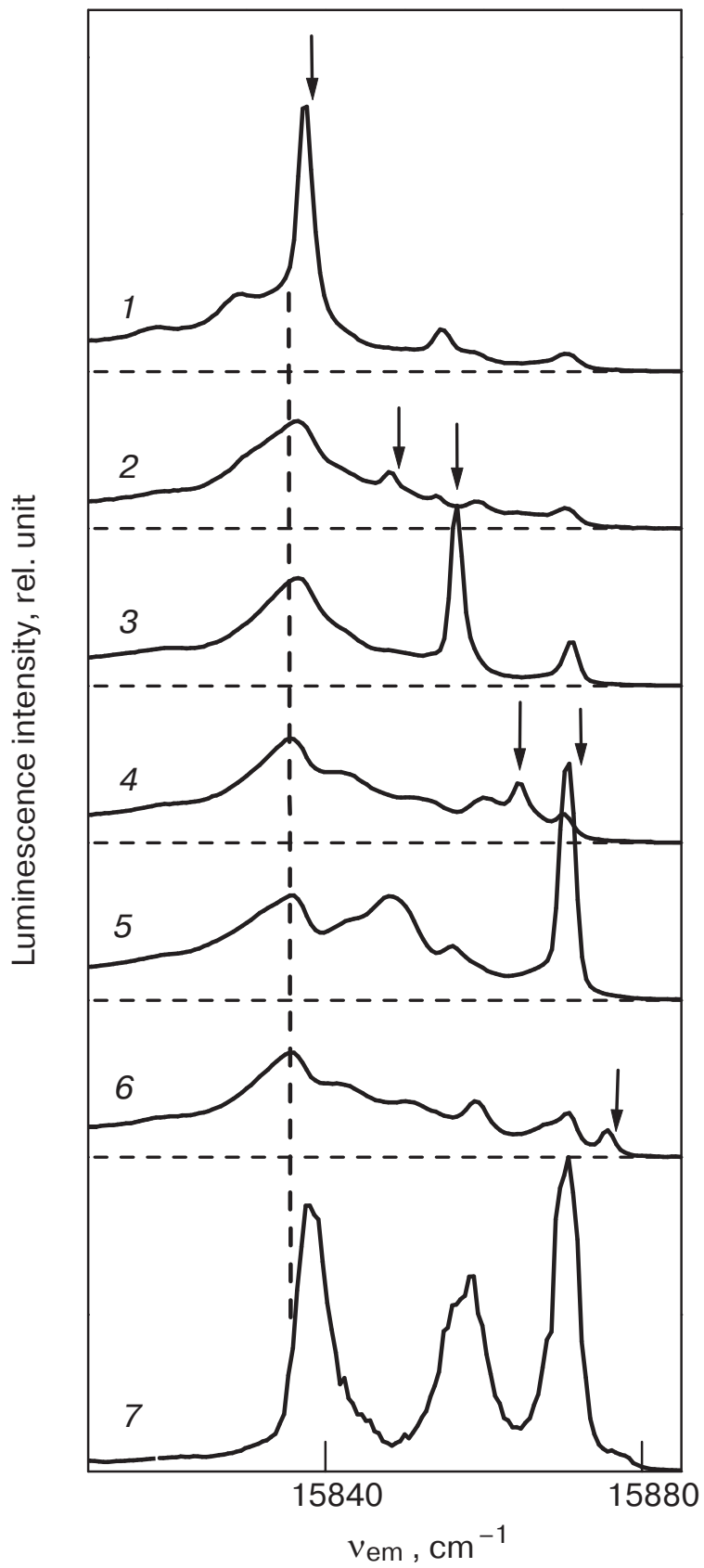

Fig. 4. Selective excitation spectra (1-6) of chlorin-doped biphenyl obtained from Fig. 1, $a$ by making horizontal cuts of $2 \mathrm{D}$ spectrum at various excitation frequencies denoted by arrows; the inhomogeneous distribution function (7) obtained from a diagonal cut of 2D spectrum taken at normal pressure. The dashed vertical line indicates the broad 2D band peak position which is independent of the excitation frequency.

tively at its emission maximum $15836 \mathrm{~cm}^{-1}$ (Fig. 2,a), repeats the triplet shape of IDF (Fig. 2,b) but is not so clear-cut, consisting of markedly wider bands. For example, while the intensity of zero-phonon lines at $15875 \mathrm{~cm}^{-1}$ is about 15 times lower as compared to the peak value at $15870 \mathrm{~cm}^{-1}$ (see Fig. 2,b), respective intensity reduction in the excitation spectrum of the 
broad 2D band is barely twofold or so (Fig. 2,a). As mentioned above, the peak position of the broad-band emission is independent of the excitation frequency; however, its spectral width exhibits some frequency-dependence. This can be seen from Fig. 1,b, where the isointensity plot is given for the excitation-dependent emission spectra, normalized to the peak value of the non-resonant broad 2D band (along the dashed horizontal line in Fig. 1,a). This band in fluorescence emission is narrowest at about $15855 \mathrm{~cm}^{-1}$ and broadens somewhat with the increase of the excitation frequency (see Fig. 1,b). Its low-energy slope, however, does not change much at frequencies $v_{\mathrm{ex}}-863.4 \mathrm{~cm}^{-1}>15865 \mathrm{~cm}^{-1}$ as follows from the parallel run of the isointensity lines. Only at the position of the blue peak in IDF some local peculiarity is observable.

In order to incorporate the above observations into the «traditional» picture of low-temperature inhomogeneously broadened impurity spectra, one has to make a number of quite unusual assumptions, such as strong quadratic electron-phonon coupling (strong deviation from the excitation-emission mirror symmetry), strong dependence of this coupling on the electronic transition energy, etc. We remind that the chlorin probe is known to have very large DebyeWaller factors (intensive zero-phonon lines with rather weak phonon sidebands) in a number of crystalline and glassy matrices. Therefore it may be more productive to seek specific for incommensurate phases novel mechanisms of relaxation. We have shown earlier [10] that in chlorin-doped biphenyl there exists an efficient mechanism of non-photochemical spectral hole burning attributed to photo-induced depinning-repinning of the incommensurate modulation wave. Such a phase shift of the modulation wave, provided it can take place not only in the excited state but also in the course of an electronic transition, may present a possible channel for formation of the broad spectral feature observed.

Due to softness of the torsional motion of two phenyl rings in biphenyl, even the dynamics in the high-pressure crystalline phase may still be non-trivial. Thus a simple interpretation of the non-resonant sideband as a phonon sideband of the resonant singlet, both seen in Fig. 3, $a$, is rather disputable because IDF of the resonant singlet is much broader than the diagonal cut (not to mention the narrower horizontal cut) of the non-resonant sideband in Fig. 3,b.

\section{Conclusions}

We have demonstrated that optical spectra of probe molecules in incommensurate and commensurate phases of biphenyl exhibit uncommon features incompatible with simple models of impurity spectra. Selective hole-burning experiments, including those at elevated pressures, may shed further light on these interesting problems.

\section{Acknowledgements}

Earlier collaboration with J. Friedrich and his group has initiated these studies. Support from the Estonian Science Foundation under grants No. 5544 and 3873 is greatly appreciated.

1. K.K. Rebane, Impurity Spectra of Solids; Elementary Theory of Vibrational Structure, Plenum Press, New York-London (1970).

2. A.Szabo, Phys. Rev. Lett. 25, 924 (1970).

3. R.I. Personov, E.I. Al'shitz, and L.A. Bykovskaya, JETP Lett. 15, 431 (1972)

4. A.A. Gorokhovskii, R.K. Kaarli, and L.A. Rebane, JETP Lett. 20, 216 (1974).

5. B.M. Kharlamov, R.I. Personov, and L.A. Bykovskaya, Opt. Commun. 12, 191 (1974).

6. K. Palewska, E.C. Meister, and U.P. Wild, J. Lumin. 50, 47 (1991).

7. R. Blinc, Phys. Rep. 79, 331 (1981).

8. A. Suisalu, V. Zazubovich, J. Kikas, J. Friebel, and J. Friedrich, Europhys. Lett. 44, 613 (1998).

9. J. Etrillard, J.E. Lasjaunias, B. Toudic, and H. Cailleau, Europhys. Lett. 38, 347 (1997).

10. V. Zazubovich, A. Suisalu, and J. Kikas, Phys. Rev. B64, 104203 (2001).

11. J. Etrillard, B. Toudic, H. Cailleau, and G. Coddens, Phys. Rev. B51, 8753 (1995).

12. V. Zazubovich, A. Suisalu, K. Leiger, A. Laisaar, An. Kuznetsov, and J. Kikas, Chem. Phys. 288, 57 (2003).

13. S.-B. Liu and M.S. Conradi, Phys. Rev. Lett. 54, 1287 (1985). 